\title{
The canonical test case for the non-commutative Singer-Wermer conjecture
}

\author{
by \\ Marc P. Thomas (Bakersfield, CA)
}

\begin{abstract}
It is a famous conjecture that every derivation on each Banach algebra leaves every primitive ideal of the algebra invariant. This conjecture is known to be true if, in addition, the derivation is assumed to be continuous. It is also known to be true if the algebra is commutative, in which case the derivation necessarily maps into the (Jacobson) radical. Because I. M. Singer and J. Wermer originally raised the question in 1955 for the case of commutative Banach algebras, the conjecture is now usually referred to as the non-commutative Singer-Wermer conjecture (the non-commutative situation being the unresolved case).

In a previous paper we demonstrated that if the conjecture fails for some non-commutative Banach algebra with discontinuous derivation, then it fails for at most finitely many primitive ideals, and each of these primitive ideals must be of finite codimension. In this paper we first show that one can make an additional reduction of any counter-example to the simplest case of a non-commutative radical Banach algebra with identity adjoined and discontinuous derivation $D$ such that $D$ does not leave the (Jacobson) radical (which is of codimension one) invariant. Second, we show that this radical Banach algebra with identity adjoined has a formal power series quotient of the form $\mathcal{A}_{0}[[t]]$ based at an element $t$ in the radical which is mapped to an invertible element by the discontinuous derivation. Finally, we specialize to the case of a separable Banach algebra and show that the pre-image of the algebra $\mathcal{A}_{0}$ is a unital subalgebra which is not an analytic set. In particular, this shows that $\mathcal{A}_{0}$ cannot be countably generated.
\end{abstract}

1. Introduction and notation. In all of the following, $\mathcal{A}$ will denote a (possibly non-commutative) Banach algebra over the complex field. It is convenient to have an identity element 1 . If $\mathcal{A}$ already has an identity element we define $\mathcal{A}^{\sharp}=\mathcal{A}$; if not, we let $\mathcal{A}^{\sharp}$ denote the algebra with identity adjoined, so that $\mathcal{A}^{\sharp} \cong \mathbb{C} \cdot 1 \oplus \mathcal{A}$.

We will let $D$ denote a derivation from $\mathcal{A}$ to itself, so that $D$ is a (possibly discontinuous) linear mapping satisfying

$$
D(a b)=a(D b)+(D a) b
$$

2000 Mathematics Subject Classification: Primary 46H05.

Key words and phrases: Banach, algebra, derivation. 
for all $a, b$ in $\mathcal{A}$. If necessary, we can extend $D$ to $\mathcal{A}^{\sharp}$ in the obvious way by defining $D(1)=0$.

We say that an ideal $P$ is primitive if it is the kernel of some (strictly) irreducible representation of the algebra $\mathcal{A}$. In 1955 I. M. Singer and J. Wermer [10] proved that a continuous derivation on a commutative Banach algebra maps into the (Jacobson) radical; they conjectured that the assumption of continuity was unnecessary. This became known as the Singer-Wermer conjecture. It is equivalent to stating that a (possibly discontinuous) derivation on a commutative Banach algebra leaves every primitive ideal invariant. This commutative case was settled in the affirmative by [12, Theorem 4.4]. Since one can ask the same question for non-commutative Banach algebras, there remained the obvious extension of the question: are all primitive ideals invariant under a derivation in every Banach algebra? This has become known as the non-commutative Singer-Wermer conjecture (the commutative case having been settled). As will become clear below, if the conjecture fails for some derivation, that derivation must be discontinuous.

We will require some results from the theory of automatic continuity. For each $n \in \mathbb{N}$ we can measure the discontinuity of each power $D^{n}$ of a derivation $D: \mathcal{A} \rightarrow \mathcal{A}$ by constructing the separating subspaces

$$
\mathcal{S}\left(D^{n}\right)=\left\{y \in \mathcal{A} \mid \exists x_{i} \rightarrow 0 \text { with } D^{n}\left(x_{i}\right) \rightarrow y\right\} .
$$

These subspaces are closed. It is a standard application of the closed graph theorem that $\mathcal{S}\left(D^{n}\right)=\{0\}$ if and only if $D^{n}$ is continuous. Furthermore, if $T$ is any continuous linear operator, it is also true that $\mathcal{S}\left(T D^{n}\right)=\overline{T \mathcal{S}\left(D^{n}\right)}$.

It follows from standard representation theory (see [7]) that primitive ideals are necessarily closed. Thus, if $P$ is a primitive ideal, we can always define the canonical continuous quotient map $Q_{P}$ from $\mathcal{A}$ to $\mathcal{A} / P$. From our remarks on automatic continuity above it follows that $Q_{P}\left(D^{n}\right)$ is continuous if and only if $\mathcal{S}\left(D^{n}\right) \subseteq P$. It is also the case [15, Lemma 1.3] that $D(P) \subseteq P$ if and only if $Q_{P}\left(D^{n}\right)$ is continuous for all $n \in \mathbb{N}$. If $D(P) \nsubseteq P$, we say that $P$ is an exceptional primitive ideal.

The following result appeared in the literature with an incorrect proof. In [13, Proposition 1.10] we gave a corrected argument.

Theorem 1.1. Let $D$ be a derivation on a Banach algebra $\mathcal{A}$. It must be the case that $Q_{P} D^{n}$ is continuous for all $n \in \mathbb{N}$ and $D(P) \subseteq P$ for all primitive ideals $P$ of $\mathcal{A}$ except possibly finitely many exceptional primitive ideals $P_{1}, \ldots, P_{k}$ which must necessarily be of finite codimension.

If the non-commutative Singer-Wermer conjecture is true then there are no exceptional primitive ideals $(k=0)$, and we are done. In this paper, we will assume that the conjecture fails for some (necessarily non-commutative) Banach algebra $\mathcal{A}$, some (necessarily discontinuous) derivation $D$, and some 
set of exceptional primitive ideals $P_{1}, \ldots, P_{k}$. We will assume in all of the following that the listing of the $P_{i}$ 's above does not contain any duplications.

In Section 2 we will reduce the above counter-example to the simplest type of counter-example, consisting of a non-commutative radical Banach algebra $\mathcal{R}$ and a new derivation $D$ which maps the radical algebra with identity adjoined into itself,

$$
D: \mathcal{R}^{\sharp} \rightarrow \mathcal{R}^{\sharp},
$$

such that $D(\mathcal{R}) \nsubseteq \mathcal{R}$, so that $\mathcal{R}$ is the only exceptional primitive ideal of $\mathcal{R}^{\sharp}$, and $\mathcal{R}$ has codimension 1 . In Section 3 we will then show that this canonical test case has a formal power series quotient based at an element $t \in \mathcal{R}$ such that $D(t)$ is invertible. In Section 4 we specialize to the case of separable Banach algebras.

2. Reduction to the case of a radical algebra with an identity adjoined. We now assume throughout that $\mathcal{A}$ is the counter-example to the Singer-Wermer conjecture that we are hypothesizing exists. Thus, $\mathcal{A}$ has all the properties specified in Theorem 1.1. For each of the exceptional primitive ideals $P_{i}$ where $i=1, \ldots, k$, choose any (strictly) irreducible representation $\pi_{i}$ with kernel $P_{i}$. Let $d_{i}$ denote the dimension (over the complex field) of the vector space on which $\pi_{i}$ operates. If $K$ is a closed ideal of $\mathcal{A}$, we let $Q_{K}$ denote the canonical continuous quotient map from $\mathcal{A}$ onto $\mathcal{A} / K$.

Definition 2.1. For each $n \in \mathbb{N}$ we denote by $\mathcal{M}_{n}$ the algebra of $n$ by $n$ matrices over the complex field.

It follows from standard representation theory (see [7]) (and from the fact that a finite-dimensional division ring which contains $\mathbb{C}$ in its center must be identical with $\mathbb{C}$ ) that for each of the exceptional primitive ideals we have the isomorphism $\mathcal{A} / P_{i} \cong \mathcal{M}_{d_{i}}$ for $i=1, \ldots, k$.

Definition 2.2. Let $\mathcal{R}$ denote the Jacobson $\operatorname{radical} \operatorname{rad}(\mathcal{A})$, of $\mathcal{A}$, and let $\mathcal{P}$ denote the intersection of all primitive ideals $P$ of $\mathcal{A}$ which are nonexceptional (i.e. $D(P) \subseteq P$ ) so that

$$
\mathcal{R}=\mathcal{P} \cap P_{1} \cap \cdots \cap P_{k} .
$$

Note that $\mathcal{P}$ is a closed two-sided ideal of $\mathcal{A}$ and that $\mathcal{P} \nsubseteq P_{i}$ for $i=$ $1, \ldots, k$ because $\mathcal{S}\left(D^{n}\right) \subseteq \mathcal{P}$ for all $n \in \mathbb{N}$.

We require a result which is due to Johnson and Sinclair [5, Lemma 3.1].

Lemma 2.3 (Johnson and Sinclair). The quotient Banach algebra

$$
\mathcal{A} /\left(P_{1} \cap \cdots \cap P_{k}\right)
$$

is finite-dimensional with the following decomposition:

$$
\mathcal{A} /\left(P_{1} \cap \cdots \cap P_{k}\right) \cong \mathcal{M}_{d_{1}} \oplus \cdots \oplus \mathcal{M}_{d_{k}} .
$$


We then have the following lemma.

Lemma 2.4. The quotient Banach algebra $\mathcal{P} / \mathcal{R}$ is finite-dimensional with the following decomposition:

$$
\mathcal{P} / \mathcal{R} \cong \mathcal{M}_{d_{1}} \oplus \cdots \oplus \mathcal{M}_{d_{k}} .
$$

Furthermore, the Jacobson radical $\mathcal{R}$ is non-trivial.

Proof. Note that

$$
\mathcal{P} / \mathcal{R}=\mathcal{P} /\left(\mathcal{P} \cap P_{1} \cap \cdots \cap P_{k}\right) \cong \frac{\mathcal{P}+\left(P_{1} \cap \cdots \cap P_{k}\right)}{P_{1} \cap \cdots \cap P_{k}} ;
$$

this is a two-sided ideal of $\mathcal{A} /\left(P_{1} \cap \cdots \cap P_{k}\right)$, which, by Lemma 2.3 , is isomorphic to $\mathcal{M}_{d_{1}} \oplus \cdots \oplus \mathcal{M}_{d_{k}}$. For each $i=1, \ldots, k$, let $\pi_{i}$ be a (strictly) irreducible representation with kernel $P_{i}$, as above. Note that $\pi_{i}(\mathcal{P})$ is a two-sided ideal of $M_{d_{i}}$, and $\pi_{i}(\mathcal{P})$ is non-trivial because $\mathcal{P} \nsubseteq P_{i}$. Since $M_{d_{i}}$ is simple, this forces $\pi_{i}(\mathcal{P})=M_{d_{i}}$ and, hence, each $\left.\pi_{i}\right|_{\mathcal{P}}$ is a (strictly) irreducible representation of $\mathcal{P}$ with kernel $\mathcal{P} \cap P_{i}$. Therefore,

$$
\frac{\mathcal{P}+\left(P_{1} \cap \cdots \cap P_{k}\right)}{P_{1} \cap \cdots \cap P_{k}}
$$

must be all of $\mathcal{A} /\left(P_{1} \cap \cdots \cap P_{k}\right)$, proving the assertion.

We note in passing that the isomorphism between $\mathcal{P} / \mathcal{R}$ and $\mathcal{M}_{d_{1}} \oplus \cdots$ $\oplus \mathcal{M}_{d_{k}}$ can be implemented via $\pi_{1} \oplus \cdots \oplus \pi_{k}$ dropped to $\mathcal{P} / \mathcal{R}$.

Now assume towards a contradiction that $\mathcal{R}=\{0\}$. This means that $\mathcal{P}$ is a finite-dimensional semisimple algebra over the complex field and so it must have an identity $e$. Since $e^{2}=e$ and since $D(\mathcal{P}) \subseteq \mathcal{P}$ it is easy to check that $D e=0$. Let $a \in \mathcal{A}$. Since $\mathcal{P}$ is a two-sided ideal we have $a e=e a e=e a$ so that $e$ is in the center of $\mathcal{A}$. We can then decompose

$$
D^{n}=e D^{n}+(1-e) D^{n}
$$

for all $n \in \mathbb{N}$. Since $\mathcal{S}\left(D^{n}\right) \subseteq \mathcal{P}$ it follows that $(1-e) \mathcal{S}\left(D^{n}\right)=\{0\}$ and, hence, that $(1-e) D^{n}$ is continuous for all $n \in \mathbb{N}$. The linear mapping $e D^{n}$ is certainly continuous when restricted to the finite-dimensional space $\mathcal{P}$ for all $n \in \mathbb{N}$. Multiplication by $e$ is a continuous projection onto the finitedimensional space $\mathcal{P}$. Since $D e=0$ it is routine to check that $e D^{n}(a)=$ $D^{n}(e a)=e D^{n}(e a)$ for all $a \in \mathcal{A}$. Consequently, $e D^{n}$ is continuous on $\mathcal{A}$ for all $n \in \mathbb{N}$. This shows that $D^{n}$ is continuous for all $n \in \mathbb{N}$ and that there could not have been any exceptional primitive ideals to begin with. Therefore, $\mathcal{R}$ cannot be trivial, ending the proof of the lemma.

We shall next show that the Jacobson radical is not invariant under the derivation. This result also follows from [4, Corollary 5.2.29] but we give the short proof here for the sake of completeness.

Lemma 2.5. There exists an element $r \in \mathcal{R}$ such that $D(r) \notin \mathcal{R}$. 
Proof. Assume towards a contradiction that $D(\mathcal{R}) \subseteq \mathcal{R}$. Since $\mathcal{R}$ is a closed two-sided ideal, the derivation $D$ then drops to a new derivation $\widehat{D}$ on the semisimple quotient Banach algebra $\mathcal{A} / \mathcal{R}$ in the usual way, namely $\widehat{D}(a+\mathcal{R})=D a+\mathcal{R}$ for $a \in \mathcal{A}$. Since $\mathcal{A} / \mathcal{R}$ is semisimple, it follows from [5, Theorem 4.1 and remarks after] or [4, Theorem 5.2.28(iii)] that $\widehat{D}$ is continuous. Since we can factor $Q_{\mathcal{R}} D^{n}$ through $\mathcal{A} / \mathcal{R}$ with

$$
Q_{\mathcal{R}} D^{n}=\widehat{D}^{n} Q_{\mathcal{R}}
$$

this shows that $Q_{\mathcal{R}} D^{n}$ is continuous for all $n \in \mathbb{N}$. But this means that $\mathcal{S}\left(D^{n}\right) \subseteq \mathcal{R} \subseteq P_{i}$ for each $n \in \mathbb{N}$ and $i=1, \ldots, k$. Obviously, this contradicts the fact that each $P_{i}$ is an exceptional primitive ideal. Therefore $D(\mathcal{R}) \nsubseteq \mathcal{R}$.

We now choose a minimal idempotent which will allow us to reduce the problem to consideration of a radical Banach algebra with identity adjoined.

Lemma 2.6. There exists a minimal (but not necessarily central) idempotent $e \in \mathcal{P}$ such that the following conditions hold:

(i) e $\mathcal{A}$ e is a closed subalgebra of $\mathcal{A}$ with identity element e whose radical is equal to $e \mathcal{R} e=e \mathcal{A} e \cap \mathcal{R}$.

(ii) eAe is a radical Banach algebra with identity adjoined and

$$
e \mathcal{A} e \cong \mathbb{C} \cdot e \oplus e \mathcal{R} e .
$$

(iii) Let $D_{1}(x)=e D(x)$ e for all $x \in e \mathcal{A} e$. Then $D_{1}$ is a derivation from eAe to itself satisfying $D_{1}(e)=0$.

(iv) There exist $t \in e \mathcal{R} e$ and $b \in e \mathcal{A} e$ such that $D_{1}(t)=e+t b, e+t b$ is invertible in $e \mathcal{A} e$, and $e-(e+t b)^{-1} \in t e \mathcal{A} e$.

Proof. We need some preliminaries. Let $x \in \mathcal{R}$ and $a \in \mathcal{A}$. Recall that $D(\mathcal{P}) \subseteq \mathcal{P}$ and note that $a D(x)=D(a x)-D(a) x$ and $D(x) a=D(x a)-$ $x D(a)$. This shows that $(D(\mathcal{R})+\mathcal{R}) / \mathcal{R}$ is a two-sided ideal in $\mathcal{P} / \mathcal{R}$ which, by Lemma 2.4, is isomorphic to $\mathcal{M}_{d_{1}} \oplus \cdots \oplus \mathcal{M}_{d_{k}}$ under the homomorphism $\pi=$ $\pi_{1} \oplus \cdots \oplus \pi_{k}$ dropped to $\mathcal{P} / \mathcal{R}$. Since $D(\mathcal{R}) \nsubseteq \mathcal{R}$ it follows that $(D(\mathcal{R})+\mathcal{R}) / \mathcal{R}$ is isomorphic to a product of some non-empty subcollection of the $M_{d_{i}}$ 's. In any case we can find some minimal idempotent, for example $e_{11}$ on the diagonal in some $M_{d_{j}}$, but zero in all other factors $M_{d_{i}}$ for $i \neq j$ in the image of $(D(\mathcal{R})+\mathcal{R}) / \mathcal{R}$ under the isomorphism $\pi$. This means that there exists $r_{1} \in \mathcal{R}$ with $\pi_{j}\left(D\left(r_{1}\right)\right)=e_{11} \in M_{d_{j}}$ and $\pi_{i}\left(D\left(r_{1}\right)\right)=0$ for $i \neq j$, so that $D\left(r_{1}\right)+\mathcal{R}$ is idempotent modulo the radical. Since $\mathcal{R}$ is both the radical of $\mathcal{A}$ and the radical of $\mathcal{P}$, applying [7, Theorem 2.3.9] or [4, Proposition 2.4.2] shows that there is an idempotent $e \in \mathcal{P}$ such that $e-D\left(r_{1}\right) \in \mathcal{R}$.

Regarding (i) and (ii), since $e \in \mathcal{P}$ it follows that $e \mathcal{A} e \subseteq \mathcal{P}$. Let $\lambda, \mu \in \mathbb{C}$ and $a, b \in \mathcal{A}$. Note that 


$$
\lambda(e a e)+\mu(e b e)=e(\lambda a e)+e(\mu b e)=e(\lambda a+\mu b) e
$$

so that $e \mathcal{A} e$ is a subspace of $\mathcal{A}$. Note that

$$
(e a e)(e b e)=e\left(a e^{2} b\right) e=e(a e b) e
$$

and

$$
e(e a e)=e^{2} a e=e a e=e a e^{2}=(e a e) e
$$

so that $e \mathcal{A} e$ is a subalgebra of $\mathcal{A}$ with identity $e$. If $\left\{a_{i}\right\}_{i=1}^{\infty}$ is any sequence in $\mathcal{A}$ and if $e a_{i} e \rightarrow b$ then $e a_{i} e=e^{2} a_{i} e^{2}=e\left(e a_{i} e\right) e \rightarrow e b e$, so that $b=e b e$, and hence $e \mathcal{A} e$ is closed. If $e a e \in e \mathcal{A} e$, apply the isomorphism $\pi$ above to obtain

$$
\pi(e a e)=\pi(e) \pi(a) \pi(e)=\lambda_{a} \pi(e)
$$

for some scalar $\lambda_{a} \in \mathbb{C}$ depending on $a$. Hence, eae $-\lambda_{a} e=x_{a} \in \mathcal{R}$, both $\lambda_{a}$ and $x_{a}$ depending on $a$. This shows that $e a e=e^{2} a e^{2}=\lambda_{a} e+e x_{a} e$. Since $a \in \mathcal{A}$ was arbitrary we have shown that

$$
e \mathcal{A} e=\mathbb{C} \cdot e+e \mathcal{R} e .
$$

If $x \in \mathcal{R} \cap e \mathcal{A} e$ then $x=e x e$. This shows that $e \mathcal{R} e=\mathcal{R} \cap e \mathcal{A} e$ and that $e \mathcal{R} e$ is a topologically nil ideal of $e \mathcal{A} e$. Since $e$ is idempotent and cannot be in the radical, this shows that the Jacobson radical of $e \mathcal{A} e$ is $e \mathcal{R} e$ and that

$$
e \mathcal{A} e \cong \mathbb{C} \cdot e \oplus e \mathcal{R} e,
$$

and we have proved (i) and (ii).

Regarding (iii), define $D_{1}(x)=e D(x) e$ for all $x \in e \mathcal{A} e$. Let $x, y \in e \mathcal{A} e$ and note that since $e$ is an identity in $e \mathcal{A} e$ we have

$$
\begin{aligned}
D_{1}(x y) & =e D(x y) e=e x D(y) e+e D(x) y e=x e D(y) e+e D(x) e y \\
& =x D_{1}(y)+D_{1}(x) y .
\end{aligned}
$$

This shows that $D_{1}$ is a derivation on $e \mathcal{A} e$. Also,

$$
D_{1}(e)=e D(e) e=e D\left(e^{2}\right) e=e^{2} D(e) e+e D(e) e^{2}=2 e D(e) e=2 D_{1}(e),
$$

which forces $D_{1}(e)=0$, and we have proved (iii).

Regarding (iv), let $r_{1}$ be as above and set $r=e r_{1} e \in e \mathcal{R} e$. We make the computation

$$
\begin{aligned}
D_{1}(r) & =D_{1}\left(e r_{1} e\right)=e D\left(e r_{1} e\right) e=e D(e) r_{1} e^{2}+e^{2} D\left(r_{1}\right) e^{2}+e^{2} r_{1} D(e) e \\
& =e D(e) r_{1} e+e D\left(r_{1}\right) e+e r_{1} D(e) e \in e+e \mathcal{R} e
\end{aligned}
$$

since $e-D\left(r_{1}\right) \in \mathcal{R}$. Hence there is $s \in e \mathcal{R} e$ such that $D_{1}(r)=e-s$. Since $e \mathcal{R} e$ is the radical of $e \mathcal{A} e$, the spectrum $\sigma(s)$ equals $\{0\}$, and $e-s$ is invertible with inverse

$$
(e-s)^{-1}=e+\sum_{j=1}^{\infty} s^{j},
$$


which is an element of $e \mathcal{A} e$. We want to make one more modification. Let $t=r(e-s)^{-1} \in e \mathcal{R} e$. We make the computation

$$
\begin{aligned}
D_{1}(t) & =D_{1}\left(r\left((e-s)^{-1}\right)\right)=(e-s)(e-s)^{-1}+r D_{1}\left((e-s)^{-1}\right) \\
& =e+r(e-s)^{-1}\left((e-s) D_{1}\left((e-s)^{-1}\right)\right) .
\end{aligned}
$$

If we define $b=(e-s) D_{1}\left((e-s)^{-1}\right)$ we have shown that $D_{1}(t)=e+t b$, which is invertible in $e \mathcal{A} e$ since $t \in e \mathcal{R} e$. Finally, consider $e-(e+t b)^{-1}$. The resolvent series is

$$
(e+t b)^{-1}=e+\sum_{j=1}^{\infty}(-1)^{j}(t b)^{j}
$$

so that

$$
e-(e+t b)^{-1}=-t \sum_{j=1}^{\infty}(-1)^{j} b(t b)^{j-1} \in t e \mathcal{A} e .
$$

The latter sum converges since $t b \in e \mathcal{R} e$ and $\sigma(t b)=\{0\}$.

From Lemma 2.6 we can immediately obtain a theorem.

TheOREM 2.7. Assume that the non-commutative Singer-Wermer conjecture fails. Then it fails for the simplest case of a non-commutative radical Banach algebra with identity adjoined. In this case the (Jacobson) radical is the only primitive ideal and it is exceptional. The corresponding (strictly) irreducible representation is the mapping of each element onto the scalar component of the identity $e$.

In order to simplify notation in the following sections, we will henceforth replace $e$ by 1 , replace $D_{1}$ by $D$, replace the radical Banach algebra $e \mathcal{R} e$ by $\mathcal{R}$ and replace the radical Banach algebra with identity adjoined $e \mathcal{A} e$ by $\mathcal{R}^{\sharp} \cong \mathbb{C} \cdot 1 \oplus \mathcal{R}$. We will continue to let $t$ denote the element in $\mathcal{R}$ with properties ensured by Lemma 2.6(iv). This will be our simplified counterexample for the remainder of the paper.

3. A formal power series quotient for the counter-example. We assume the simplified counter-example of the previous section guaranteed by Lemma 2.6. Our goal in this section is to obtain an algebraic decomposition of $\mathcal{R}^{\sharp}$ in the form of a formal power series quotient (see [14, Definition 2.1 and remarks following]). We wish to stress that we will use the terms subalgebra, ideal, and subspace in the algebraic sense, that is, we do not assume that these substructures are closed.

For $x, y \in \mathcal{R}^{\sharp}$ let $[x, y]=x y-y x$ be the usual bracket notation for a commutator. Since $\mathcal{R}^{\sharp} \cong \mathbb{C} \cdot 1 \oplus \mathcal{R}$ it is routine to show that the components of the identity will be eliminated and that $[x, y] \in \mathcal{R}$. 
Definition 3.1. Let $\mathcal{C}_{0}$ denote the subspace generated by the commutators in $\mathcal{R}$, so that

$$
\mathcal{C}_{0} \equiv\left\{\sum_{i=1}^{n} \alpha_{i}\left[x_{i}, y_{i}\right] \mid n \in \mathbb{N},\left\{\alpha_{i}\right\}_{i=1}^{n} \subseteq \mathbb{C},\left\{x_{i}\right\}_{i=1}^{n},\left\{y_{i}\right\}_{i=1}^{n} \subseteq \mathcal{R}\right\} .
$$

Definition 3.2. Let $\mathcal{C}$ denote the two-sided ideal generated by the commutators.

Since $[a x, y]=a[x, y]+[a, y] x$ it easily follows that one needs to form either only the left or only the right products, for example

$$
\mathcal{C}=\left\{\sum_{i=1}^{n} a_{i}\left[x_{i}, y_{i}\right] \mid n \in \mathbb{N},\left\{a_{i}\right\}_{i=1}^{n} \subseteq \mathcal{R}^{\sharp},\left\{x_{i}\right\}_{i=1}^{n},\left\{y_{i}\right\}_{i=1}^{n} \subseteq \mathcal{R}\right\} .
$$

It is also clear that $\mathcal{C} \subseteq \mathcal{R}$. In general, neither $\mathcal{C}_{0}$ nor $\mathcal{C}$ is closed.

Lemma 3.3. The two-sided ideal $\mathcal{C}$ generated by the commutators is invariant under the derivation $D$.

Proof. It is a routine computation using the properties of a derivation to show that $D([x, y])=[D(x), y]+[x, D(y)]$. From this, it easily follows that $D(\mathcal{C}) \subseteq \mathcal{C}$.

Our goal is to produce a formal power series quotient based at the element $t$ from Lemma 2.6(iv) of the previous section. To do this we will need to factor the non-commutative Banach algebra $\mathcal{R}^{\sharp}$ by some ideal which will necessarily have to contain all commutators and all elements of infinite height in $t$.

Definition 3.4. Using our element $t \in \mathcal{R}$ from the previous section, let $\mathcal{C}_{t}$ denote the set constructed as follows:

$$
\mathcal{C}_{t} \equiv \bigcap_{n=1}^{\infty}\left(t^{n} \mathcal{R}^{\sharp}+\mathcal{C}\right) .
$$

Lemma 3.5. The set $\mathcal{C}_{t}$ is a two-sided ideal of $\mathcal{R}^{\sharp}$ contained in $\mathcal{R}$ and is invariant under the derivation $D$.

Proof. It is obvious that $\mathcal{C}_{t}$ is a right ideal and that it is invariant under left multiplication by $t$. Let $a, b \in \mathcal{R}^{\sharp}$ and $n \in \mathbb{N}$. Since

$$
a t^{n} b=t^{n} a b+\left[a, t^{n}\right] b \in t^{n} a b+\mathcal{C}
$$

it is clear that $\mathcal{C}_{t}$ is also a left ideal. Since $t \in \mathcal{R}$ and $\mathcal{C} \subseteq \mathcal{R}$ it is clear that $\mathcal{C}_{t} \subseteq \mathcal{R}$. Finally, we claim that for all $n \in \mathbb{N}$ we have

$$
D\left(t^{n}\right) \in t^{n-1} \mathcal{R}^{\sharp}+\mathcal{C} .
$$

This is obvious for $n=1$ since $t^{0}=1$. Suppose that we have shown that

$$
D\left(t^{n}\right)=t^{n-1} a_{n}+c_{n}
$$


for some $a_{n} \in \mathcal{R}^{\sharp}$ and $c_{n} \in \mathcal{C}$. Then

$$
D\left(t^{n+1}\right)=t D\left(t^{n}\right)+D(t) t^{n}=t^{n} a_{n}+t c_{n}+t^{n} D(t)+\left[D(t), t^{n}\right] \in t^{n} \mathcal{R}^{\sharp}+\mathcal{C} .
$$

By induction the assertion holds for all $n \in \mathbb{N}$ and from this and Lemma 3.3 it easily follows that

$$
D\left(t^{n} \mathcal{R}^{\sharp}+\mathcal{C}\right) \subseteq t^{n-1} \mathcal{R}^{\sharp}+\mathcal{C}
$$

for all $n \in \mathbb{N}$. Therefore $D\left(\mathcal{C}_{t}\right) \subseteq \mathcal{C}_{t}$.

We now have a commutative unital quotient algebra $\mathcal{R}^{\sharp} / \mathcal{C}_{t}$. Since $\mathcal{C}_{t}$ is not in general closed, this quotient is not in general a Banach algebra.

Definition 3.6. If $\mathcal{A}_{0}$ is a commutative unital algebra, let $\mathcal{A}_{0}[[x]]$ denote the algebra of formal power series in the indeterminate $x$ with coefficients in $\mathcal{A}_{0}$, thus

$$
\mathcal{A}_{0}[[x]] \equiv\left\{\sum_{n=0}^{\infty} a_{n} x^{n} \mid\left\{a_{n}\right\}_{n=0}^{\infty} \subseteq \mathcal{A}_{0}\right\} .
$$

Our next goal is to find a unital subalgebra $\mathcal{A}_{0}$ of $\mathcal{R}^{\sharp} / \mathcal{C}_{t}$ such that $\mathcal{R}^{\sharp} / \mathcal{C}_{t} \cong \mathcal{A}_{0}[[t]]$. Before we can do this we need to develop a natural $p$-adic topology on $\mathcal{R}^{\sharp} / \mathcal{C}_{t}$, and this will require several preliminary results.

Definition 3.7. Let $L_{t}$ denote the continuous linear operator of left multiplication by $t$ on $\mathcal{R}^{\sharp}$. Let $\mathcal{L}_{t}$ denote the largest $L_{t}$-divisible subspace of $\mathcal{R}^{\sharp}$, that is,

$$
\left(\lambda-L_{t}\right) \mathcal{L}_{t}=\mathcal{L}_{t}
$$

for all $\lambda \in \mathbb{C}$ and $\mathcal{L}_{t}$ is the largest subspace of $\mathcal{R}^{\sharp}$ with this property.

Remarks. Since $t \in \mathcal{R}$ and $t \mathcal{L}_{t}=\mathcal{L}_{t}$, it follows that $\mathcal{L}_{t} \subseteq \mathcal{R}$. Since $\lambda \neq 0$ forces $\lambda-L_{t}$ to be bijective, one only needs to check that $L_{t} \mathcal{L}_{t}=\mathcal{L}_{t}$.

Divisible subspaces play a prominent role in the theory of automatic continuity (see [9] or [4]). We next need a condition first introduced by G. R. Allan in [1].

Definition 3.8. We say that $t$ has finite closed descent if there exists $m_{0} \in \mathbb{N}$ such that

$$
t^{m_{0}} \in \overline{\mathcal{L}_{t}} .
$$

REMARKs. This is equivalent to requiring that $t^{m_{0}} \in \overline{t^{m_{0}+1} \mathcal{R} \sharp}$ since then

$$
\overline{t^{m_{0}} \mathcal{R}^{\sharp}}=\overline{\overline{t^{m_{0}} \mathcal{R}^{\sharp}}}=\overline{L_{t} \overline{t^{m_{0}} \mathcal{R}^{\sharp}}} .
$$

Hence, $L_{t}$ acts densely on $\overline{t^{m_{0}} \mathcal{R}^{\sharp}}$ and, by the Mittag-Leffler theorem ([2, Section 3, Théorème 1] or $\left[4\right.$, A.1.24, A.1.25]), it follows that $\overline{t^{m_{0}} \mathcal{R}^{\sharp}}$ must contain a dense $L_{t}$-divisible subspace $X$ which must be contained in the largest $L_{t}$-divisible subspace $\mathcal{L}_{t}$. Hence $t^{m_{0}} \in \overline{\mathcal{L}_{t}}$.

Recall the definition of separating subspaces from Section 1. 
Definition 3.9. Define $I_{1}=\mathcal{S}(D)$ and for $k \geq 2$ define

$$
I_{k}=\overline{\mathcal{S}(D)+\mathcal{S}\left(D^{2}\right)+\cdots+\mathcal{S}\left(D^{k}\right)} .
$$

We note that $\mathcal{S}\left(D^{k}\right)$ by itself may not be an ideal if $k \geq 2$. However, the following holds.

Lemma 3.10. The sequence $\left\{I_{k}\right\}_{k=1}^{\infty}$ is a non-decreasing family of closed two-sided ideals in $\mathcal{R}^{\sharp}$.

Proof. It is certainly clear that all $I_{k}$ are closed subspaces of $\mathcal{R}^{\sharp}$. Hence we may form the continuous linear canonical quotient map $Q_{I_{k}}$ from $\mathcal{R}^{\sharp}$ to the Banach space $\mathcal{R}^{\sharp} / I_{k}$ for each $k \in \mathbb{N}$. We need to show that these closed subspaces are ideals.

It is routine to show that $I_{1}$ is a closed two-sided ideal since the linear mappings $D(a \cdot)-a D(\cdot)$ and $D(\cdot a)-D(\cdot) a$ are continuous for all $a \in \mathcal{R}^{\sharp}$. Suppose we have shown that $I_{1}, \ldots, I_{k-1}$ are all closed two-sided ideals, and consider $I_{k}$. Let $b \in \mathcal{S}\left(D^{k}\right)$. Hence, there exist $a_{n} \rightarrow 0$ with $D^{k}\left(a_{n}\right) \rightarrow b$. We will observe the convention that $D^{0}=I$ in the following. Let $a \in \mathcal{R}^{\sharp}$ be fixed and note that

$$
a D^{k}\left(a_{n}\right)=D^{k}\left(a a_{n}\right)-\sum_{l=0}^{k-1}\left(\begin{array}{c}
k \\
l
\end{array}\right) D^{k-l}(a) D^{l}\left(a_{n}\right) .
$$

Since $I_{k-1}$ is a closed two-sided ideal containing $\mathcal{S}\left(D^{l}\right)$ for $l=1, \ldots, k-1$, it follows that

$$
Q_{I_{k-1}}\left(D^{k-l}(a) \mathcal{S}\left(D^{l}\right)\right)=Q_{I_{k-1}}\left(D^{k-l}(a)\right) Q_{I_{k-1}}\left(\mathcal{S}\left(D^{l}\right)\right)=\{0\}
$$

for $l=1, \ldots, k-1$. Since $I_{k} \supseteq I_{k-1}$ it also follows that

$$
Q_{I_{k}}\left(D^{k-l}(a) \mathcal{S}\left(D^{l}\right)\right)=\{0\}
$$

for $l=1, \ldots, k-1$, although we do not yet know that $I_{k}$ is an ideal or that $Q_{I_{k}}$ is multiplicative. In any case we have shown that $Q_{I_{k}} D^{k-l}(a) D^{l}(\cdot)$ is a continuous linear mapping for $l=1, \ldots, k-1$. It is also clearly continuous if $l=0$. Since $a_{n} \rightarrow 0$ and $D^{k}\left(a_{n}\right) \rightarrow b$, applying $Q_{I_{k}}$ to the equation above yields

$$
Q_{I_{k}}(a b)=\lim _{n \rightarrow \infty} Q_{I_{k}}\left(a D^{k}\left(a_{n}\right)\right)=\lim _{n \rightarrow \infty} Q_{I_{k}}\left(D^{k}\left(a a_{n}\right)\right)+0 .
$$

Since $a a_{n} \rightarrow 0$ and $I_{k} \supset \mathcal{S}\left(D^{k}\right)$ it follows that $Q_{I_{k}} D^{k}$ is continuous and that

$$
Q_{I_{k}}(a b)=\lim _{n \rightarrow \infty} Q_{I_{k}}\left(D^{k}\left(a a_{n}\right)\right)=0 .
$$

This shows that $a b \in I_{k}$. Since $a$ was arbitrary in $\mathcal{R}^{\sharp}$ we have shown that

$$
\mathcal{R}^{\sharp} \mathcal{S}\left(D^{k}\right) \subseteq I_{k}=\overline{I_{k-1}+\mathcal{S}\left(D^{k}\right)} .
$$

Since $I_{k-1}$ is a two-sided ideal, this shows that $I_{k}$ must be a left ideal. An analogous argument shows that $I_{k}$ must be a right ideal, and induction completes the proof of the lemma. 
REMARK. We have actually shown a bit more, namely that

$$
Q_{I_{k-1}} D^{k}(a \cdot)-Q_{I_{k-1}} a D^{k}(\cdot) \text { is continuous for } k=2,3, \ldots
$$

For our next result we require a standard theorem (adjusted to our situation) from the theory of automatic continuity ([13, Proposition 1.3] or [4, Theorem 5.2.5]) which we state here for reference.

THEOREM 3.11. Let $\left\{T_{i}\right\}_{i=1}^{\infty}$ be a sequence of continuous linear operators defined from $\mathcal{R}^{\sharp}$ to itself and let $\left\{R_{i}\right\}_{i=1}^{\infty}$ be a sequence of continuous linear operators with domain $\mathcal{R}^{\sharp}$ (but which may map onto other Banach spaces). Let $S$ be a (possibly discontinuous) linear map from $\mathcal{R}^{\sharp}$ to itself. If

$$
R_{n} S T_{1} T_{2} \cdots T_{m} \text { is continuous for } m>n \text {, }
$$

then

$$
R_{n} S T_{1} T_{2} \cdots T_{n} \text { is continuous for all sufficiently large } n \text {. }
$$

In the theory of automatic continuity the following result would be referred to as a stability lemma. Let $\mathbb{Z}^{+}$denote the non-negative integers.

Lemma 3.12. For each $k \in \mathbb{N}$ there exists $n_{k} \in \mathbb{Z}^{+}$such that

$$
t^{n_{k}} I_{k} \subseteq \overline{\mathcal{L}_{t}} \quad \text { and } \quad \overline{t^{n} I_{k}}=\overline{t^{n_{k}} I_{k}} \quad \text { for all } n \geq n_{k} .
$$

Proof. If $k=1$, this is the classical stability lemma of automatic continuity (see [9] or [4, Theorem 5.2.5(iii)]) since $I_{1}=\mathcal{S}(D)$ and $D L_{t}-L_{t} D$ is continuous. Hence there exists $n_{1} \in \mathbb{Z}^{+}$such that

$$
\overline{t^{n} \mathcal{S}(D)}=\overline{t^{n_{1}} \mathcal{S}(D)}
$$

for $n \geq n_{1}$ and therefore

$$
\overline{t t^{n_{1}} \mathcal{S}(D)}=\overline{t^{n_{1}} \mathcal{S}(D)} .
$$

The Mittag-Leffler theorem ([2, Section 3, Théorème 1] or [4]) and consideration of the projective limit

$$
\lim _{\longleftarrow}\left(\overline{t^{n_{1}} \mathcal{S}(D)}, L_{t}\right)
$$

show that $\overline{t^{n_{1}} \mathcal{S}(D)}$ contains a dense $L_{t}$-divisible subspace which must, of course, be contained in the largest $L_{t}$-divisible subspace $\mathcal{L}_{t}$, hence

$$
t^{n_{1}} \mathcal{S}(D) \subseteq \overline{\mathcal{L}_{t}} .
$$

Let $k \geq 2$. Assume towards a contradiction that we have found $n_{1}, \ldots, n_{k-1}$ such that

$$
t^{n_{l}} I_{l} \subseteq \overline{\mathcal{L}_{t}} \quad \text { and } \quad \overline{t^{n} I_{l}}=\overline{t^{n_{l}} I_{l}} \quad \text { whenever } n \geq n_{l}
$$

for $l=1, \ldots, k-1$. Suppose that the result fails for $k$. It then follows that

$$
\overline{t^{n} I_{k}} \supset \overline{t^{n+1} I_{k}} \supseteq \overline{t^{n+1} I_{k-1}}=\overline{t^{n_{k-1} I_{k-1}}}
$$


for $n \geq n_{k-1}$, where $\supset$ indicates "proper" containment. Let $n \geq n_{k-1}$, let $m \geq n$, and consider the map

$$
\left(Q_{\overline{t^{n+1} I_{k}}} t^{n_{k-1}}\right) \circ D^{k} \circ L_{t}^{m-n_{k-1}} .
$$

Let $a \in \mathcal{R}^{\sharp}$ and note that

$$
\begin{aligned}
& Q_{\overline{t^{n+1} I_{k}}}\left(t^{n_{k-1}} D^{k}\left(t^{m-n_{k-1}} a\right)\right) \\
& =Q_{t^{n+1} I_{k}}\left(t^{m} D^{k}(a)\right)+Q_{t^{n+1} I_{k}}\left(t^{n_{k-1}} \sum_{l=0}^{k-1}\left(\begin{array}{c}
k \\
l
\end{array}\right) D^{k-l}\left(t^{m-n_{k-1}}\right) D^{l}(a)\right) .
\end{aligned}
$$

Since $m \geq n \geq n_{k-1}$ we have

$$
t^{n_{k-1}} D^{k-l}\left(t^{m-n_{k-1}}\right) \mathcal{S}\left(D^{l}\right) \subseteq t^{n_{k-1}} I_{k-1} \subseteq \overline{t^{n+1} I_{k}},
$$

which shows that the terms in the sum for $l=0,1, \ldots, k-1$ are continuous in $a$. Therefore

$$
Q_{\bar{t}^{n+1} I_{k}}\left(t^{n_{k-1}} D^{k}\left(t^{m-n_{k-1}} a\right)\right)-Q_{\bar{t}^{n+1} I_{k}}\left(t^{m} D^{k}(a)\right)
$$

is continuous in $a$. Suppose that $Q_{\overline{t^{n+1} I_{k}}}\left(t^{n} \mathcal{S}\left(D^{k}\right)\right)=\{0\}$. Then $t^{n} \mathcal{S}\left(D^{k}\right) \subseteq$ $\overline{t^{n+1} I_{k}}$ and since $I_{k}=\overline{I_{k-1}+\mathcal{S}\left(D^{k}\right)}$ it follows that

$$
t^{n} I_{k} \subseteq \overline{t^{n} I_{k-1}+\overline{t^{n+1} I_{k}}} \subseteq \overline{\overline{t^{n-1} I_{k-1}}}+\overline{t^{n+1} I_{k}} \subseteq \overline{t^{n+1} I_{k}},
$$

a contradiction. Therefore $Q_{\overline{t^{n+1} I_{k}}}\left(t^{n} \mathcal{S}\left(D^{k}\right)\right) \neq\{0\}$, whence $Q_{\overline{t^{n+1} I_{k}}} t^{n} D^{k}$ is discontinuous. Since $Q_{\overline{t^{n+1} I_{k}}} t^{m} D^{k}$ is clearly continuous if $m \geq n+1$, we have shown that

$Q_{\bar{t}^{n+1} I_{k}} t^{m} D^{k}$ is discontinuous if $m=n$ and continuous if $m>n$.

Using the continuity of the difference above, we see that

$$
\left(Q_{t^{n+1} I_{k}} t^{n_{k-1}}\right) \circ D^{k} \circ L_{t}^{m-n_{k-1}} \text { is }\left\{\begin{array}{l}
\text { discontinuous if } m=n, \\
\text { continuous if } m>n
\end{array}\right.
$$

This contradicts the standard theorem from the theory of automatic continuity as stated in Theorem 3.11 with $S=D^{k}, T_{i}=L_{t}$ or $I$, and $R_{n}=\left(Q_{\overline{t^{n+1} I_{k}}} t^{n_{k-1}}\right)$. Hence, the result holds for all $k \in \mathbb{N}$ and there does exist $n_{k} \in \mathbb{Z}^{+}$such that

$$
\overline{t^{n} I_{k}}=\overline{t^{n_{k}} I_{k}} \quad \text { for all } n \geq n_{k} .
$$

In addition, it is clear that

$$
\overline{\overline{t^{n_{k} I_{k}}}}=\overline{t^{n_{k} I_{k}}},
$$

and another application of the Mittag-Leffler theorem shows that $\overline{t^{n_{k}} I_{k}}$ contains a dense $L_{t}$-divisible subspace which must, of course, be contained in the largest $L_{t}$-divisible subspace $\mathcal{L}_{t}$. Therefore

$$
t^{n_{k}} I_{k} \subseteq \overline{\mathcal{L}_{t}}
$$

and induction finishes the proof of the lemma. 
We are now able to prove that $t$ has finite closed descent even in this non-commutative setting.

Proposition 3.13. The element $t$ has finite closed descent, that is, there exists $m_{0} \in \mathbb{N}$ such that

$$
t^{m_{0}} \in \overline{\mathcal{L}_{t}}
$$

Proof. For each $n \in \mathbb{N}$ define

$$
\mathcal{E}_{n}=\left\{a \in \mathcal{R}^{\sharp} \mid t^{n} a \in \overline{\mathcal{L}_{t}}\right\}
$$

Since $\mathcal{L}_{t}$ is invariant under left multiplication by $t$ and is a right ideal it follows that its closure has these same properties. It is then routine to check that each $\mathcal{E}_{n}$ is invariant under left multiplication by $t$ and is a closed right ideal. Define

$$
\mathcal{E}=\bigcup_{n=1}^{\infty} \mathcal{E}_{n} .
$$

Again, it is routine to check that $\mathcal{E}$ is invariant under left multiplication by $t$ and is a right ideal (not necessarily closed). By Lemma 3.12, $I_{k} \subseteq \mathcal{E}_{n_{k}}$ for each $k \in \mathbb{N}$, and therefore

$$
\mathcal{E} \supseteq \bigcup_{k=1}^{\infty} I_{k} .
$$

Suppose that $\mathcal{E} \subseteq \mathcal{R}$. Since the radical $\mathcal{R}$ is closed, for every $k \in \mathbb{N}$ we have $I_{k} \subseteq \mathcal{E} \subseteq \overline{\mathcal{E}} \subseteq \overline{\mathcal{R}}$. Since $\mathcal{S}\left(D^{k}\right) \subseteq I_{k}$ it follows that $Q_{\mathcal{R}} D^{k}$ is continuous for every $k \in \mathbb{N}$. But $\mathcal{R}$ is a primitive ideal, so by [15, Lemma 1.3] this would force $D(\mathcal{R}) \subseteq \mathcal{R}$, a contradiction. We therefore conclude that $\mathcal{E} \nsubseteq \mathcal{R}$. Consequently, there is an invertible element of the form $1+x$ in $\mathcal{E}$ with $x \in \mathcal{R}$. Since $\mathcal{E}$ is a union, there must exist $m_{0} \in \mathbb{N}$ such that $1+x \in \mathcal{E}_{m_{0}}$. Since $\mathcal{E}_{m_{0}}$ is a right ideal this means that $1=(1+x)(1+x)^{-1} \in \mathcal{E}_{m_{0}}$. Therefore, $t^{m_{0}}=t^{m_{0}} \cdot 1 \in \overline{\mathcal{L}_{t}}$, ending the proof of the proposition.

We can now put a natural p-adic topology $\tau_{t}$ on the quotient space $\mathcal{R}^{\sharp} / \mathcal{C}_{t}$ with a local base at zero consisting of the sets of cosets

$$
\left\{t^{n} \mathcal{R}^{\sharp}+\mathcal{C}_{t}\right\}_{n=1}^{\infty} \text {. }
$$

We will call this the Zariski topology (see [16] or [14, Section 3, discussion]). We stress that $\left(\mathcal{R}^{\sharp} / \mathcal{C}_{t}, \tau_{t}\right)$ is not a locally convex topological vector space; a priori, $\mathcal{C}_{t}$ might even be dense in $\mathcal{R}$. However, we will show that $\left(\mathcal{R}^{\sharp} / \mathcal{C}_{t}, \tau_{t}\right)$ is a complete metric space if we pick any translation invariant metric $d(\cdot, \cdot)$ which generates the topology $\tau_{t}$. For our purposes the following will suffice:

$$
d\left(x+\mathcal{C}_{t}, y+\mathcal{C}_{t}\right)=2^{-n} \quad \text { if } x-y \in t^{n} \mathcal{R}^{\sharp}+\mathcal{C} \text { and } x-y \notin t^{n+1} \mathcal{R}^{\sharp}+\mathcal{C} .
$$

Note that we are tacitly using the fact that $t^{n} \mathcal{R}^{\sharp}+\mathcal{C}=t^{n} \mathcal{R}^{\sharp}+\mathcal{C}_{t}$. 
We have the following proposition which essentially shows absolute summability.

Proposition 3.14. Let $m_{0}$ be the natural number guaranteed in Proposition 3.13. Given any sequence $\left\{a_{n}\right\}_{n=m_{0}}^{\infty} \subseteq \mathcal{R}^{\sharp}$ there exists $a \in \overline{\mathcal{L}_{t}}$ such that

$$
a-\sum_{m=m_{0}}^{M} t^{m} a_{m} \in t^{M-m_{0}+1} \mathcal{R}^{\sharp} \quad \text { for all } M \geq m_{0} .
$$

Proof. This argument, which appeals to the finite closed descent of $t$, was first used by Allan in [1]. First note that if $m \in \mathbb{N}$ with $m \geq m_{0}$ and $x \in \mathcal{R}^{\sharp}$ then $t^{m} x \in \overline{\mathcal{L}_{t}}$ since $\overline{\mathcal{L}_{t}}$ is a right ideal. Consider the projective system

$\lim _{(}\left(\overline{\mathcal{L}_{t}}, A_{n}\right) \quad$ with $A_{n}(x)=t x+t^{m_{0}} a_{m_{0}+n} \quad$ for $x \in \overline{\mathcal{L}_{t}}$ and $n \in \mathbb{Z}^{+}$.

It is routine to check that each map $A_{n}$ is affine, continuous on $\overline{\mathcal{L}_{t}}$, and, since $t \mathcal{L}_{t}=\mathcal{L}_{t}$, has dense range in $\overline{\mathcal{L}_{t}}$ for $n=0,1,2, \ldots$ The Mittag-Leffler theorem ([2, Section 3, Théorème 1] or [4]) and consideration of the above projective limit show that there exists an element $a \in \overline{\mathcal{L}_{t}}$ and elements $\left\{x_{j}\right\}_{j=1}^{\infty} \subseteq \overline{\mathcal{L}_{t}}$ such that

$$
a=A_{0}\left(x_{1}\right), \quad x_{1}=A_{1}\left(x_{2}\right), \quad x_{2}=A_{2}\left(x_{3}\right), \quad \ldots
$$

From the above equations we compute

$$
\begin{aligned}
a & =A_{0}\left(x_{1}\right)=t x_{1}+t^{m_{0}} a_{m_{0}}=t\left(A_{1}\left(x_{2}\right)\right)+t^{m_{0}} a_{m_{0}} \\
& =t\left(x_{2}+t^{m_{0}} a_{m_{0}+1}\right)+t^{m_{0}} a_{m_{0}}=t^{2} x_{2}+t^{m_{0}+1} a_{m_{0}+1}+t^{m_{0}} a_{m_{0}}=\cdots \\
& =t^{M-m_{0}+1} x_{M-m_{0}+1}+\sum_{m=m_{0}}^{M} t^{m} a_{m} \quad \text { for all } M \geq m_{0},
\end{aligned}
$$

establishing the assertion of the proposition.

COROLlary 3.15. The commutative unital algebra and topological space $\left(\mathcal{R}^{\sharp} / \mathcal{C}_{t}, \tau_{t}\right)$ endowed with the translation invariant metric $d(\cdot, \cdot)$ is a complete metric space.

Proof. Going back to the definitions early in this section we note that $\mathcal{C}_{t}$ is an ideal containing the ideal $\mathcal{C}$ generated by the commutators. Hence $\mathcal{R}^{\sharp} / \mathcal{C}_{t}$ is a commutative algebra. Let a "dot" denote the coset in $\mathcal{R}^{\sharp} / \mathcal{C}_{t}$. It is clear that $\dot{i}$ is an identity for $\mathcal{R}^{\sharp} / \mathcal{C}_{t}$. To show the completeness of $\tau_{t}$ as determined by the metric $d(\cdot, \cdot)$ it will suffice to show that every series of the form $\sum_{m=0}^{\infty} \dot{t}^{m} \dot{a}_{m}$ in $\mathcal{R}^{\sharp} / \mathcal{C}_{t}$ converges. Note that

$$
\sum_{m=0}^{\infty} \dot{t}^{m} \dot{a}_{m}=\sum_{m=0}^{m_{0}-1} \dot{t}^{m} \dot{a}_{m}+\sum_{m=m_{0}}^{\infty} \dot{t}^{m} \dot{a}_{m} .
$$


Since the finite sum on the left is convergent and since Proposition 3.14 guarantees the convergence of the sum on the right, completeness of $\tau_{t}$ follows.

We will continue to use a "dot" to denote the coset in $\mathcal{R}^{\sharp} / \mathcal{C}_{t}$. Since $D\left(\mathcal{C}_{t}\right) \subseteq \mathcal{C}_{t}$ we can drop $D$ to a derivation $\widehat{D}$ on $\mathcal{R}^{\sharp} / \mathcal{C}_{t}$ as follows:

$$
\widehat{D}(\dot{a}) \equiv(\dot{D(a)}) \quad \text { for } a \in \mathcal{R}^{\sharp} .
$$

It is mildly annoying that $\widehat{D}(\dot{t})=\dot{1}+\dot{t} \dot{b}$. We would prefer that $\widehat{D}(\dot{t})=\dot{1}$, and we will be able to show later (see Theorem 3.18) that this is possible by "modifying" $t$ (multiplying it by a suitable unit) once we have proved that $\mathcal{R}^{\sharp} / \mathcal{C}_{t}$ has a formal power series decomposition. For now, we can only modify $\widehat{D}$ by defining

$$
\dot{D}(\dot{a})=(1+\dot{t} b)^{-1} \widehat{D}(\dot{a}) \quad \text { for } \dot{a} \in \mathcal{R}^{\sharp} / \mathcal{C}_{t} .
$$

Hence $\dot{D}(\dot{t})=\dot{1}$, but we warn the reader that $\dot{D}$ is only a derivation on the commutative algebra $\mathcal{R}^{\sharp} / \mathcal{C}_{t}$ and not in general on $\mathcal{R}^{\sharp}$ (due to its noncommutativity).

We are now able to show that $\mathcal{R}^{\sharp}$ has a formal power series quotient based at $t$ (see [14, Definition 2.1 and remarks following]). The proof of this proceeds in exactly the same manner as that of [12, Proposition 2.18 to Proposition 2.24], using the properties of the derivation $\dot{D}$ and the completeness of $\tau_{t}$. This result is also essentially [16, Theorem 4] moved to a Banach algebra setting.

TheOREM 3.16. The commutative unital algebra and topological space $\left(\mathcal{R}^{\sharp} / \mathcal{C}_{t}, \tau_{t}\right)$ endowed with the translation invariant metric $d(\cdot, \cdot)$ is a complete metric space with derivation $\dot{D}$, which is continuous with respect to $\tau_{t}$. There exists an element $\dot{t}$ satisfying $\dot{D}(\dot{t})=\dot{1}$, the identity of $\mathcal{R}^{\sharp} / \mathcal{C}_{t}$. In addition:

(i) Define the map

$$
\Theta(\dot{a})=e^{-\dot{t} \dot{D}}(\dot{a})=\dot{a}-\dot{t} \dot{D}(\dot{a})+\frac{\dot{t}^{2}}{2 !} \dot{D}^{2}(\dot{a})-\frac{\dot{t}^{3}}{3 !} \dot{D}^{3}(\dot{a})+\cdots
$$

It follows that $\Theta$ is a $\tau_{t}$-continuous homomorphism and projection onto a unital subalgebra $\mathcal{A}_{0}$ of $\mathcal{R}^{\sharp} / \mathcal{C}_{t}$ and $\mathcal{A}_{0}$ is identical to the null space of $\dot{D}$. For all $\dot{a} \in \mathcal{R}^{\sharp} / \mathcal{C}_{t}$ we have $(I-\Theta)(\dot{a}) \in \dot{t}\left(\mathcal{R}^{\sharp} / \mathcal{C}_{t}\right)$.

(ii) (Formal power series quotient based at $t$ ) There is an algebraic isomorphism $\mathcal{R}^{\sharp} / \mathcal{C}_{t} \cong \mathcal{A}_{0}[[t]]$ which takes $\dot{t}$ to the indeterminate $t$ of $\mathcal{A}_{0}[[t]]$. The map $\Theta$ is the projection onto the first term of the series.

(iii) The action of $\dot{D}$ on $\mathcal{R}^{\sharp} / \mathcal{C}_{t}$ is essentially formal differentiation. Let $\dot{a}_{n} \in \mathcal{A}_{0}$ for $n \in \mathbb{Z}^{+}$. Then

$$
\dot{D}\left(\sum_{n=0}^{\infty} \dot{t}^{n} \dot{a}_{n}\right)=\sum_{n=1}^{\infty} n \dot{t}^{n-1} \dot{a}_{n} .
$$


Proof. The derivation $\dot{D}$ is continuous with respect to the Zariski topology $\tau_{t}$ because $\dot{D}\left(\dot{t}^{n}\left(\mathcal{R}^{\sharp} / \mathcal{C}_{t}\right)\right) \subseteq \dot{t}^{n-1}\left(\mathcal{R}^{\sharp} / \mathcal{C}_{t}\right)$. It is routine to check that $\Theta$ is a homomorphism satisfying $\dot{D}\left(\Theta\left(\mathcal{R}^{\sharp} / \mathcal{C}_{t}\right)\right)=\{0\}$. The homomorphism $\Theta$ is $\tau_{t}$-continuous because the identity map $I$ and the derivation $\dot{D}$ are $\tau_{t}$-continuous. Let $\mathcal{A}_{0}$ be the image of $\Theta$. Again, it is routine to check that $\mathcal{A}_{0}$ is a unital subalgebra of $\mathcal{R}^{\sharp} / \mathcal{C}_{t}$ which is contained in the null space of $\dot{D}$. But if $\dot{a} \in \mathcal{R}^{\sharp} / \mathcal{C}_{t}$ and $\dot{D}(\dot{a})=0$, it is also clear that $\Theta(\dot{a})=\dot{a}$. Therefore, $\mathcal{A}_{0}$ is identical to the null space of $\dot{D}$. Since $\Theta$ is $\tau_{t}$-continuous and since $\Theta(\dot{t})=0$ it follows that $\Theta^{2}=\Theta$, so that $\Theta$ is both a homomorphism and projection onto $\mathcal{A}_{0}$. Let $\dot{a} \in \mathcal{R}^{\sharp} / \mathcal{C}_{t}$ and compute

$$
(I-\Theta)(\dot{a})=\dot{t} \dot{D}(\dot{a})-\frac{\dot{t}^{2}}{2 !} \dot{D}^{2}(\dot{a})+\frac{\dot{t}^{3}}{3 !} \dot{D}^{3}(\dot{a})+\cdots \in \dot{t}\left(\mathcal{R}^{\sharp} / \mathcal{C}_{t}\right) .
$$

This proves (i).

It is routine to show that every $\dot{a} \in \mathcal{R}^{\sharp} / \mathcal{C}_{t}$ has a series expansion of the form $\sum_{n=0}^{\infty} \dot{t}^{n} \dot{a}_{n}$ where $\dot{a}_{n} \in \mathcal{A}_{0}$, as follows. Note that

$$
\dot{a}=\Theta(\dot{a})+(I-\Theta)(\dot{a})=\dot{a}_{0}+\dot{t}_{1}
$$

for some $\dot{a}_{0} \in \mathcal{A}_{0}$ and $\dot{b}_{1} \in \mathcal{R}^{\sharp} / \mathcal{C}_{t}$. Then

$$
\dot{b}_{1}=\Theta\left(\dot{b}_{1}\right)+(I-\Theta)\left(\dot{b}_{1}\right)=\dot{a}_{1}+\dot{t}_{2}
$$

for some $\dot{a}_{1} \in \mathcal{A}_{0}$ and $\dot{b}_{2} \in \mathcal{R}^{\sharp} / \mathcal{C}_{t}$. Then

$$
\dot{b}_{2}=\Theta\left(\dot{b}_{2}\right)+(I-\Theta)\left(\dot{b}_{2}\right)=\dot{a}_{2}+\dot{t}_{3}
$$

for some $\dot{a}_{2} \in \mathcal{A}_{0}$ and $\dot{b}_{3} \in \mathcal{R}^{\sharp} / \mathcal{C}_{t}$. Continue this process to obtain sequences $\left\{\dot{a}_{n}\right\}_{n=0}^{\infty} \subseteq \mathcal{A}_{0}$ and $\left\{\dot{b}_{n}\right\}_{n=1}^{\infty} \subseteq \mathcal{R}^{\sharp} / \mathcal{C}_{t}$. Substituting for the $b_{i}$ 's one obtains the equation $\dot{a}=\sum_{n=0}^{\infty} \dot{t}^{n} \dot{a}_{n}$.

Conversely, every such series in $\mathcal{R}^{\sharp} / \mathcal{C}_{t}$ must converge in $\tau_{t}$. Since $\mathcal{A}_{0}$ is an algebra, the product of two series is the formal product.

It remains to show uniqueness. Suppose that $\left\{\dot{a}_{n}\right\}_{n=0}^{\infty} \subseteq \mathcal{A}_{0}$ and $0=$ $\sum_{n=0}^{\infty} \dot{t}^{n} \dot{a}_{n}$. Assume that the terms of the series are not all zero and let $\dot{a}_{n_{0}}$ be the first non-zero term. Then we have

$$
0=\dot{t}^{n_{0}}\left(\dot{a}_{n_{0}}+\sum_{n=n_{0}+1}^{\infty} \dot{t}^{n-n_{0}} \dot{a}_{n}\right) .
$$

Apply $\Theta \circ \dot{D}^{n_{0}}$ to the above equation, noting that $\Theta(\dot{t})=0$ to obtain

$$
0=n_{0} !\left(\Theta\left(\dot{a}_{n_{0}}\right)\right) \text {. }
$$

But $\Theta\left(\dot{a}_{n_{0}}\right)=\dot{a}_{n_{0}}$ since $\Theta$ is a projection onto $\mathcal{A}_{0}$ and $\dot{a}_{n_{0}} \in \mathcal{A}_{0}$. This contradication shows that all terms of the series must have been zero, and establishes (ii).

Finally, the proof of (iii) is a straightforward computation using the $\tau_{t}$-continuity of the derivation $\dot{D}$ and the fact that $\mathcal{A}_{0}$ is the null space of $\dot{D}$. 
Let $\dot{a}_{n} \in \mathcal{A}_{0}$ for $n \in \mathbb{Z}^{+}$. Then

$$
\dot{D}\left(\sum_{n=0}^{\infty} \dot{t}^{n} \dot{a}_{n}\right)=\sum_{n=0}^{\infty} \dot{D}\left(\dot{t}^{n} \dot{a}_{n}\right)=\sum_{n=0}^{\infty}\left(n \dot{t}^{n-1} \dot{a}_{n}+\dot{t}^{n} \dot{D}\left(\dot{a}_{n}\right)\right)=\sum_{n=1}^{\infty} n \dot{t}^{n-1} \dot{a}_{n} .
$$

Now that we have the formal power series decomposition we can go back to Lemma 2.6 and show that we could have chosen $t$ so that $b \in \mathcal{C}_{t}$. We first require a lemma.

Lemma 3.17. If $s \in \mathcal{R}$ and $u$ is invertible in $\mathcal{R}^{\sharp}$ with $s=$ tu then

$$
\mathcal{C}_{s} \equiv \bigcap_{n=1}^{\infty}\left(s^{n} \mathcal{R}^{\sharp}+\mathcal{C}\right)=\bigcap_{n=1}^{\infty}\left(t^{n} \mathcal{R}^{\sharp}+\mathcal{C}\right)=\mathcal{C}_{t} .
$$

Proof. Note that the ideal $\mathcal{C}$ generated by the commutators is contained in both $\mathcal{C}_{s}$ and $\mathcal{C}_{t}$. Let $n \in \mathbb{N}$ and note that

$$
s^{n} \mathcal{R}^{\sharp}=(t u)^{n} \mathcal{R}^{\sharp} \subseteq t^{n} u^{n} \mathcal{R}^{\sharp}+\mathcal{C} \subseteq t^{n} \mathcal{R}^{\sharp}+\mathcal{C} .
$$

Exchanging the roles of $s$ and $t$ and noting that $t=s u^{-1}$ we also obtain

$$
t^{n} \mathcal{R}^{\sharp} \subseteq s^{n} \mathcal{R}^{\sharp}+\mathcal{C},
$$

from which it follows that $s^{n} \mathcal{R}^{\sharp}+\mathcal{C}=t^{n} \mathcal{R}^{\sharp}+\mathcal{C}$. Since this holds for all $n \in \mathbb{N}$, we have $\mathcal{C}_{s}=\mathcal{C}_{t}$, ending the proof of the lemma.

TheOREM 3.18. There exists an invertible element $u \in \mathcal{R}^{\sharp}$ such that if $s=$ tu then $\mathcal{C}_{s}=\mathcal{C}_{t}$ and

$$
D(s)=1+s b \quad \text { with } b \in \mathcal{C}_{t}=\mathcal{C}_{s}
$$

so that $\widehat{D}(\dot{s})=\dot{1}$. In addition, $1-(1+s b)^{-1} \in s \mathcal{R}^{\sharp}$. Finally, the conclusion of Theorem 3.16 holds with $\dot{D}$ replaced by $\widehat{D}$ and $t$ replaced by $s$.

Proof. From Theorem 3.16 we have $\mathcal{R}^{\sharp} / \mathcal{C}_{t} \cong \mathcal{A}_{0}[[t]]$ and the action of $\dot{D}=(1+\dot{t} b)^{-1} \widehat{D}$ is simply formal differentiation on $\mathcal{R}^{\sharp} / \mathcal{C}_{t}$. Expand

$$
(1+\dot{t} b)^{-1}=\dot{1}+\sum_{n=1}^{\infty} \dot{t}^{n} \dot{b}_{n}
$$

for some $\left\{b_{n}\right\}_{n=1}^{\infty} \subseteq \mathcal{A}_{0}$. Note that the leading term will be $\dot{1}$ because of Lemma 2.6(iv), which shows that $1-(1+t b)^{-1} \in t \mathcal{R}^{\sharp}$. Pick any element $u_{1} \in \mathcal{R}^{\sharp}$ satisfying

$$
\dot{u}_{1}=\dot{1}+\sum_{n=1}^{\infty} \frac{\dot{t}^{n}}{n+1} \dot{b}_{n} .
$$

It is clear that $u_{1}$ is invertible since $t$ is in the radical $\mathcal{R}$ of $\mathcal{R}^{\sharp}$. Let $s_{1}=t u_{1}$ and compute

$$
\dot{s}_{1}=\dot{t}+\sum_{n=1}^{\infty} \frac{\dot{t}^{n+1}}{n+1} \dot{b}_{n}
$$


Hence,

$$
(1+\dot{t} b)^{-1} \widehat{D}\left(\dot{s}_{1}\right)=(1+\dot{t} b)^{-1} .
$$

This shows that $\widehat{D}\left(\dot{s}_{1}\right)=\dot{1}$ and, hence, that $D\left(s_{1}\right)=1+c$ for some $c \in \mathcal{C}_{t}$. We want to make one additional change in $s_{1}$. Note that $(1+c)^{-1}=1+d$ for some $d \in \mathcal{R}$ satisfying $c+d+c d=0$. Since $\mathcal{C}_{t}$ is a two-sided ideal of $\mathcal{R}^{\sharp}$ this shows that $d \in \mathcal{C}_{t}$ also. Now let $s=s_{1}(1+d)=t u_{1}(1+d)$. Then

$$
D(s)=D\left(s_{1}(1+d)\right)=(1+c)(1+d)+s_{1} D(1+d)=1+s(1+c) D(d) .
$$

Since $D\left(\mathcal{C}_{t}\right) \subseteq \mathcal{C}_{t}$ it is clear that $(1+c) D(d) \in \mathcal{C}_{t}$. Let $u=u_{1}(1+d)$, which is invertible in $\mathcal{R}^{\sharp}$ so that $s=t u$ and $\mathcal{C}_{s}=\mathcal{C}_{t}$. Let $b=(1+c) D(d) \in \mathcal{C}_{s}$ and note that we have

$$
D(s)=1+s b \quad \text { so that } \widehat{D}(\dot{s})=\dot{1} .
$$

Finally, since $s b \in \mathcal{R}$ note that

$$
1-(1+s b)^{-1}=1-\sum_{n=0}^{\infty}(-1)^{n}(s b)^{n}=s \sum_{n=1}^{\infty}(-1)^{n} b(b s)^{n-1} \in s \mathcal{R}^{\sharp} .
$$

The rest is immediate, and this ends the proof of the theorem.

4. Separable Banach algebras and analytic subsets. Here we specialize to the case of separable Banach algebras and we require some standard topological results from the theory of Polish and analytic spaces. A very thorough reference is [3], which contains much more than the small number of results we use below. All of the results we require can also be found in [4]. We will assume throughout this section that $\mathcal{R}$ is a separable Banach algebra.

First, some definitions:

(4.1a) A topological space $E$ is called Polish if it has a metric $\mu$ under which it is a complete separable metric space.

(4.1b) A topological space $F$ is called analytic if it is the continuous image of some Polish space.

The following are standard, well-known results about analytic sets:

(4.1c) Open and closed subsets of an analytic space are also analytic.

(4.1d) Countable unions and countable intersections of analytic spaces are analytic.

(4.1e) The continuous image of an analytic space is analytic.

(4.1f) Provided the mapping is continuous, the preimage of an analytic set is analytic.

The following are standard, well-known results about analytic sets in a locally convex topological vector space (LCTVS): 
(4.1g) In a LCTVS the span and convex hull of an analytic set are analytic; hence the sum of two analytic subspaces is analytic.

(4.1h) If $X$ is a non-meager LCTVS, and $Y$ and $Z$ are two analytic subspaces such that $X \cong Y \oplus Z$ as algebraic vector spaces, then $Y$ and $Z$ are closed and the direct sum is topological.

(4.1i) (Pettis Lemma) If $F$ is any analytic non-meager subset of a LCTVS then $F-F$ is a neighborhood of the zero vector.

Recall Theorems 3.16/3.18, which ensure the existence of a power series decomposition of the quotient algebra

$$
\mathcal{R}^{\sharp} / \mathcal{C}_{t} \cong \mathcal{A}_{0}[[t]] .
$$

Let $\mathcal{A}=\left\{x \in \mathcal{R}^{\sharp} \mid D(x) \in \mathcal{C}_{t}\right\}$. We also showed that $\mathcal{A}_{0} \cong \mathcal{A} / \mathcal{C}_{t}$, so that $\mathcal{A}$ is the preimage of $\mathcal{A}_{0}$ under the canonical quotient map $Q: \mathcal{R}^{\sharp} \rightarrow \mathcal{R}^{\sharp} / \mathcal{C}_{t}$.

If $\mathcal{R}$ is separable, it is easy to use the (4.1) results above and show that $\mathcal{C}_{0}, \mathcal{C}$, and $\mathcal{C}_{t}$ are all analytic subsets of $\mathcal{R}$. However, the quotient algebra $\mathcal{R}^{\sharp} / \mathcal{C}_{t}$ is not a Banach algebra because the ideal $\mathcal{C}_{t}$, although an analytic set, is not in general closed.

Proposition 4.1. Let $\mathcal{A}$ be the preimage of $\mathcal{A}_{0}$ under the quotient map $Q: \mathcal{R}^{\sharp} \rightarrow \mathcal{R}^{\sharp} / \mathcal{C}_{t} \cong \mathcal{A}_{0}[[t]]$. Then $\mathcal{A}$ is not an analytic subset of $\mathcal{R}^{\sharp}$.

Proof. Assume towards a contradiction that $\mathcal{A}$ is analytic. Let $\pi$ denote the continuous projection of $\mathcal{R}^{\sharp}$ onto $\mathcal{R}$. Let $\mathcal{B}=\pi(\mathcal{A})$. Since $\mathcal{A}$ is analytic, so is $\mathcal{B}$. It is clear that $\mathcal{A} \cong \mathbb{C} \cdot 1 \oplus \mathcal{B}$ and that $1 \notin \mathcal{B}$. Now, for every $n \in \mathbb{N}$,

$$
\mathcal{B}+t \mathcal{B}+t^{2} \mathcal{B}+\cdots+t^{n} \mathcal{B} \text { is analytic. }
$$

Hence

$$
\mathcal{B}+t \mathcal{B}+t^{2} \mathcal{B}+\cdots+t^{n} \mathcal{B}+t^{n+1} \mathcal{R}^{\sharp}+\mathcal{C}_{t} \text { is analytic. }
$$

But $\mathcal{R}^{\sharp}$ has the following direct sum decomposition:

$$
\mathcal{R}^{\sharp} \cong \operatorname{span}\left\{1, t, t^{2}, \ldots, t^{n}\right\} \oplus\left(\mathcal{B}+t \mathcal{B}+t^{2} \mathcal{B}+\cdots+t^{n} \mathcal{B}+t^{n+1} \mathcal{R}^{\sharp}+\mathcal{C}_{t}\right)
$$

and the first subspace, being finite-dimensional, is closed and, hence, analytic. By $(4.1 \mathrm{~h})$ this forces the decomposition to be topological, and

$$
\mathcal{B}+t \mathcal{B}+t^{2} \mathcal{B}+\cdots+t^{n} \mathcal{B}+t^{n+1} \mathcal{R}^{\sharp}+\mathcal{C}_{t} \text { is also closed. }
$$

Hence, for each $n \in \mathbb{N}$ the projection $\pi_{n}$ onto $\operatorname{span}\left\{1, t, t^{2}, \ldots, t^{n}\right\}$ is bounded by some constant $K_{n}$. This is incompatible with a power series decomposition as follows. Choose an element $a \in \mathcal{R}$ whose power series representation in $\mathcal{R}^{\sharp} / \mathcal{C}_{t} \cong \mathcal{A}_{0}[[t]]$ is $\sum_{n=1}^{\infty} \lambda_{n} t^{n}$ where each $\lambda_{n}$ is chosen to satisfy

$$
\left|\lambda_{n}\right|>n\left(K_{n}+K_{n-1}\right) /\left\|t^{n}\right\| \text {. }
$$


We then compute

$$
\begin{aligned}
K_{n}\|a\| & \geq\left\|\pi_{n}(a)\right\|=\left\|\lambda_{n} t^{n}+\sum_{i=0}^{n-1} \lambda_{i} t^{i}\right\| \geq\left|\lambda_{n}\right|\left\|t^{n}\right\|-\left\|\sum_{i=0}^{n-1} \lambda_{i} t^{i}\right\| \\
& \geq\left|\lambda_{n}\right|\left\|t^{n}\right\|-K_{n-1}\|a\| .
\end{aligned}
$$

Solving for $\|a\|$, we see that

$$
\|a\| \geq\left|\lambda_{n}\right|\left\|t^{n}\right\| /\left(K_{n}+K_{n-1}\right) \geq n
$$

for each $n \in \mathbb{N}$. This shows that $a$ has infinite norm, a contradiction, and proves that $\mathcal{A}$ could not have been analytic.

Proposition 4.2. The algebra $\mathcal{A}_{0}$ is not countably generated.

Proof. We leave the finitely generated case to the reader. Assume $\mathcal{A}_{0}$ is countably generated by $\left\{\dot{a}_{i}\right\}_{i=1}^{\infty}$. Start with $\mathcal{A}_{1}$ being the algebra generated by $\mathcal{C}_{t}$ and $a_{1}$. Since $\mathcal{C}_{t}$ is an analytic set, so is $\mathcal{A}_{1}$. If $\mathcal{A}_{n}$ has been generated and is analytic, let $\mathcal{A}_{n+1}$ be the algebra generated by $\mathcal{A}_{n}$ and the element $a_{n+1}$. It will also be an analytic set. Induction shows that each $\mathcal{A}_{k}$ for $k=1,2, \ldots$ is an analytic set. Therefore, the subalgebra

$$
\mathcal{A}=\bigcup_{k=1}^{\infty} \mathcal{A}_{k}
$$

is also analytic by (4.1d). This contradicts Proposition 4.1 and shows that $\mathcal{A}_{0}$ could not have been countably generated.

If one believes that the non-commutative Singer-Wermer conjecture is true, an immediate question is: what additional conditions would force $\mathcal{A}$ to be an analytic set? If one believes that there is a counter-example, one might try to find a non-commutative Banach algebra which has a formal power series quotient in which $\mathcal{A}_{0} \cong \mathbb{C}\left[\left[t_{1}, t_{2}, \ldots\right]\right]$, the algebra of formal power series in infinitely many commuting variables so that

$$
\mathcal{R}^{\sharp} / \mathcal{C}_{t} \cong \mathbb{C}\left[\left[t, t_{1}, t_{2}, \ldots\right]\right] .
$$

Charles Read in [6] has constructed both commutative and non-commutative Fréchet algebras of this type which have discontinuous derivations mapping out of the radical. It is not clear, however, if his construction can be extended to build a counter-example which is a Banach algebra, since $t$ would have to have finite closed descent.

\section{References}

[1] G. R. Allan, Embedding the algebra of all formal power series in a Banach algebra, Proc. London Math. Soc. (3) 25 (1972), 329-340. 


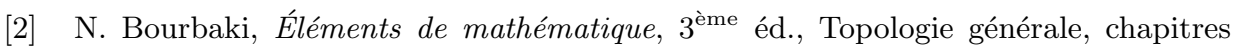
I-II, Actualités Sci. Indust. 1142, Hermann, Paris, 1961.

[3] J. P. R. Christensen, Topology and Borel Structure, North-Holland, Amsterdam, 1974.

[4] H. G. Dales, Banach Algebras and Automatic Continuity, London Math. Soc. Monogr. (N.S.) 24, Oxford Univ. Press, Oxford, 2000.

[5] B. E. Johnson and A. M. Sinclair, Continuity of derivations and a problem of Kaplansky, Amer. J. Math. 90 (1968), 1067-1073.

[6] C. J. Read, Derivations on Fréchet algebras with large separating subspace, Proc. Amer. Math. Soc. 130 (2002), 3671-3677.

[7] C. E. Rickart, General Theory of Banach Algebras, Krieger, 1974.

[8] V. Runde, Range inclusion results for derivations on noncommutative Banach algebras, Studia Math. 105 (1993), 159-172.

[9] A. M. Sinclair, Automatic Continuity of Linear Operators, London Math. Soc. Lecture Note Ser. 21, Cambridge Univ. Press, Cambridge, 1976.

[10] I. M. Singer and J. Wermer, Derivations on commutative normed algebras, Math. Ann. 129 (1955), 260-264.

[11] M. P. Thomas, Automatic continuity for linear functions intertwining continuous linear operators on Fréchet spaces, Canad. J. Math. 30 (1978), 518-530.

[12] - The image of a derivation is contained in the radical, Ann. of Math. 128 (1988), 435-460.

[13] - Primitive ideals and derivations on non-commutative Banach algebras, Pacific J. Math. 159 (1993), 139-152.

[14] - Local power series quotients of commutative Banach and Fréchet algebras, Trans. Amer. Math. Soc. 355 (2003), 2135-2160.

[15] -, Reduction of discontinuity for derivations on Fréchet algebras, J. London Math. Soc. 76 (2007), 165-180.

[16] O. Zariski, Studies in equisingularity I: Equivalent singularities of plane algebroid curves, Amer. J. Math. 87 (1965), 507-536.

Department of Mathematics and Computer Science

California State University at Bakersfield

9001 Stockdale Hwy.

Bakersfield, CA 93311-1022, U.S.A.

E-mail:marc@cs.csubak.edu 